\title{
Solvability of Minimal Graph Equation Under Pointwise \\ Pinching Condition for Sectional Curvatures
}

\section{Casteras, Jean-Baptiste}

2017-04

Casteras , J-B , Heinonen , E \& Holopainen , I 2017 , ' Solvability of Minimal Graph Equation Under Pointwise Pinching Condition for Sectional Curvatures ' , Journal of Geometric Analysis , vol. 27 , no. 2 , pp. 1106-1130 . https://doi.org/10.1007/s12220-016-9712-0

http://hdl.handle.net/10138/214841

https://doi.org/10.1007/s12220-016-9712-0

acceptedVersion

Downloaded from Helda, University of Helsinki institutional repository.

This is an electronic reprint of the original article.

This reprint may differ from the original in pagination and typographic detail.

Please cite the original version. 


\title{
SOLVABILITY OF MINIMAL GRAPH EQUATION UNDER POINTWISE PINCHING CONDITION FOR SECTIONAL CURVATURES
}

\author{
JEAN-BAPTISTE CASTERAS, ESKO HEINONEN, AND ILKKA HOLOPAINEN
}

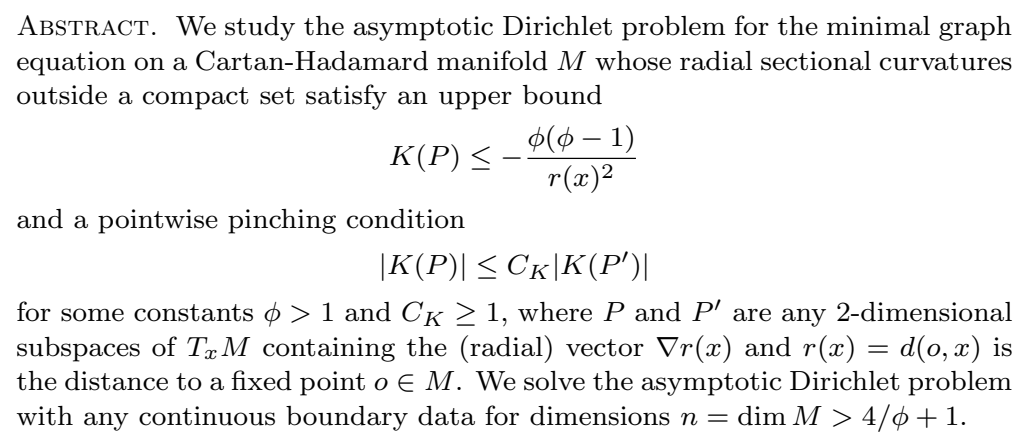

\section{InTRODUCTION}

In this paper we are interested in the asymptotic Dirichlet problem for minimal graph equation

$$
\operatorname{div} \frac{\nabla u}{\sqrt{1+|\nabla u|^{2}}}=0
$$

on a Cartan-Hadamard manifold $M$ of dimension $n \geq 2$. We recall that a CartanHadamard manifold is a simply connected complete Riemannian manifold with non-positive sectional curvature. Since the exponential map $\exp _{o}: T_{o} M \rightarrow M$ is a diffeomorphism for every point $o \in M$, it follows that $M$ is diffeomorphic to $\mathbb{R}^{n}$. One can define an asymptotic boundary $\partial_{\infty} M$ of $M$ as the set of all equivalence classes of unit speed geodesic rays on $M$. Then the compactification of $M$ is given by $\bar{M}=M \cup \partial_{\infty} M$ equipped with the cone topology. We also notice that $\bar{M}$ is homeomorphic to the closed Euclidean unit ball; for details, see [16].

The asymptotic Dirichlet problem on $M$ for some operator $\mathcal{Q}$ is the following: Given a function $f \in C\left(\partial_{\infty} M\right)$ does there exist a (unique) function $u \in C(\bar{M})$ such that $\mathcal{Q}[u]=0$ on $M$ and $u \mid \partial_{\infty} M=f$ ? We will consider this problem for the minimal graph operator (or the mean curvature operator) appearing in (1.1). It is also worth noting that a function $u$ satisfies 11.1) if and only if the graph $\{(x, u(x)): x \in M\}$ is a minimal hypersurface in the product space $M \times \mathbb{R}$.

The asymptotic Dirichlet problem on Cartan-Hadamard manifolds has been solved for various operators and under various assumptions on the manifold. The first result for this problem was due to Choi [7] when he solved the asymptotic Dirichlet problem for the Laplacian assuming that the sectional curvature has a negative upper bound $K_{M} \leq-a^{2}<0$, and that any two points at infinity can be

2000 Mathematics Subject Classification. Primary 58J32; Secondary 53C21.

Key words and phrases. minimal graph equation, Dirichlet problem, Hadamard manifold.

J.-B.C. supported by MIS F.4508.14 (FNRS).

E.H. supported by the Academy of Finland, project 252293 and the Wihuri Foundation.

I.H. supported by the Academy of Finland, project 252293. 
separated by convex neighborhoods. Anderson [1 showed that such convex sets exist provided the sectional curvature of the manifold satisfies $-b^{2} \leq K_{M} \leq-a^{2}<0$. We point out that Sullivan [29] solved independently the asymptotic Dirichlet problem for the Laplacian under the same curvature assumptions but using probabilistic arguments. Cheng 6] was the first to solve the problem for the Laplacian under the same type of pointwise pinching assumption for the sectional curvatures as we consider in this paper. Later the asymptotic Dirichlet problem has been generalized for $p$-harmonic and $\mathcal{A}$-harmonic functions under various curvature assumptions, see [4, 21, 23], 30], 31.

Concerning the mean curvature operator, there has been a growing interest in developing a theory of constant (or prescribed) mean curvature hypersurfaces in Riemannian manifolds. For instance, Guan and Spruck [19] investigated the problem of finding complete hypersurfaces of constant mean curvature with prescribed asymptotic boundaries at infinity in the hyperbolic space (see also the recent [20] and references therein). On the other hand, Dajczer, Hinojosa, and de Lira ([12], [10, [11]) have studied Killing graphs of prescribed mean curvature under curvature conditions on the ambient space. Further studies include so-called half-space theorems in product spaces $M \times \mathbb{R}_{+}$; see [26], [13], and references therein. In these investigations, a priori gradient estimates based on the classical maximum principle for elliptic equations are indispensable. To motivate further the study of the asymptotic Dirichlet problem for the minimal graph equation, we recall the papers [8] and [17] by Collin, Gálvez, and Rosenberg who were able to construct harmonic diffeomorphisms from the complex plane $\mathbb{C}$ onto the hyperbolic plane $\mathbb{H}^{2}$ and onto any Hadamard surface $M$ whose curvature is bounded from above by a negative constant, respectively, hence disproving a conjecture of Schoen and Yau [27]. The key idea in their constructions was to solve the Dirichlet problem on unbounded ideal polygons with boundary values $\pm \infty$ on the sides of the ideal polygons.

Concerning the asymptotic Dirichlet problem for the equation (1.1), Casteras, Holopainen, and Ripoll studied the problem under curvature bounds

$$
-b(r(x))^{2} \leq K(P) \leq-a(r(x))^{2},
$$

where $a, b:[0, \infty) \rightarrow[0, \infty)$ are smooth functions subject to some growth conditions. Here and throughout the paper $r(x)=d(x, o)$ stands for the distance to a fixed point $o \in M$. As special cases of their main theorem [3. Theorem 1.6] we state here the following two solvability results.

Theorem 1.1. 3. Theorem 1.5, Corollary 1.7] Let $M$ be a Cartan-Hadamard manifold of dimension $n \geq 2$. Suppose that

$$
-r(x)^{2(\phi-2)-\varepsilon} \leq K(P) \leq-\frac{\phi(\phi-1)}{r(x)^{2}}
$$

or

$$
-r(x)^{-2-\varepsilon} e^{2 k r(x)} \leq K(P) \leq-k^{2}
$$

for some constants $\varepsilon>0, \phi>1$, and $k>0$, and for all 2-dimensional subspaces $P \subset T_{x} M$, with $x \in M \backslash B\left(o, R_{0}\right)$. Then the asymptotic Dirichlet problem for (1.1) is uniquely solvable for any boundary data $f \in C\left(\partial_{\infty} M\right)$.

The solvability of the asymptotic Dirichlet problem for (1.1) under curvature assumptions (1.3) was earlier obtained by Ripoll and Telichevesky in 25; see also 14 and [15. Recently, Casteras, Holopainen, and Ripoll 44 were able to weaken the curvature upper bound to an almost optimal one. 
Theorem 1.2. 4, Theorem 5] Let $M$ be a Cartan-Hadamard manifold of dimension $n \geq 3$ satisfying the curvature assumption

$$
-\frac{(\log r(x))^{2 \bar{\varepsilon}}}{r(x)^{2}} \leq K(P) \leq-\frac{1+\varepsilon}{r(x)^{2} \log r(x)}
$$

for some constants $\varepsilon>\bar{\varepsilon}>0$ and for any 2-dimensional subspace $P \subset T_{x} M$, with $x \in M \backslash B\left(o, R_{0}\right)$. Then the asymptotic Dirichlet problem for 11.1 is uniquely solvable for any boundary data $f \in C\left(\partial_{\infty} M\right)$.

It is worth noting that even a strict negative curvature upper bound alone is not sufficient in dimensions $n \geq 3$ for the solvability of the asymptotic Dirichlet problem for (1.1). Indeed, in 22. Holopainen and Ripoll generalized Borbély's counterexample [2] to cover the minimal graph equation.

Our main theorem is the following. It is worth noticing that no lower bounds for sectional curvatures are needed. Instead we assume a pointwise pinching condition on sectional curvatures.

Theorem 1.3. Let $M$ be a Cartan-Hadamard manifold of dimension $n \geq 2$ and let $\phi>1$. Assume that

$$
K(P) \leq-\frac{\phi(\phi-1)}{r(x)^{2}},
$$

where $K(P)$ is the sectional curvature of any two-dimensional subspace $P \subset T_{x} M$ containing the radial vector $\nabla r(x)$, with $x \in M \backslash B\left(o, R_{0}\right)$. Suppose also that there exists a constant $C_{K}<\infty$ such that

$$
|K(P)| \leq C_{K}\left|K\left(P^{\prime}\right)\right|
$$

whenever $x \in M \backslash B\left(o, R_{0}\right)$ and $P, P^{\prime} \subset T_{x} M$ are two-dimensional subspaces containing the radial vector $\nabla r(x)$. Moreover, suppose that the dimension $n$ and the constant $\phi$ satisfy the relation

$$
n>\frac{4}{\phi}+1
$$

Then the asymptotic Dirichlet problem for the minimal graph equation (1.1) is uniquely solvable for any boundary data $f \in C\left(\partial_{\infty} M\right)$.

We notice that if we choose the constant $\phi$ in the curvature assumption to be bigger than 4 , then our theorem will hold in every dimension $n \geq 2$. Similarly, if we let the dimension $n$ to be at least 5 , we can take the constant $\phi$ to be as close to 1 as we wish.

In this paper we will proceed as follows. Section 2 is devoted to preliminaries. We will recall some facts about Cartan-Hadamard manifolds, Jacobi equations, the minimal graph equation and Young functions. In Section 3 we will prove our main theorem i.e. the solvability of the minimal graph equation under the curvature assumptions (1.4), (1.5) and (1.6). We will adopt the strategies used in [4, 6], 30] and 31 .

\section{Preliminaries}

2.1. Cartan-Hadamard manifolds. Recall that a Cartan-Hadamard manifold is a complete and simply connected Riemannian manifold with non-positive sectional curvature. Let $M$ be a Cartan-Hadamard manifold and $\partial_{\infty} M$ the sphere at infinity, then we denote $\bar{M}=M \cup \partial_{\infty} M$. The sphere at infinity is defined as the set of all equivalence classes of unit speed geodesic rays in $M$; two such rays $\gamma_{1}$ and $\gamma_{2}$ are equivalent if

$$
\sup _{t \geq 0} d\left(\gamma_{1}(t), \gamma_{2}(t)\right)<\infty
$$


The equivalence class of $\gamma$ is denoted by $\gamma(\infty)$. For each $x \in M$ and $y \in \bar{M} \backslash\{x\}$ there exists a unique unit speed geodesic $\gamma^{x, y}: \mathbb{R} \rightarrow M$ such that $\gamma^{x, y}(0)=x$ and $\gamma^{x, y}(t)=y$ for some $t \in(0, \infty]$. For $x \in M$ and $y, z \in \bar{M} \backslash\{x\}$ we denote by

$$
\varangle_{x}(y, z)=\varangle\left(\dot{\gamma}_{0}^{x, y}, \dot{\gamma}_{0}^{x, z}\right)
$$

the angle between vectors $\dot{\gamma}_{0}^{x, y}$ and $\dot{\gamma}_{0}^{x, z}$ in $T_{x} M$. If $v \in T_{x} M \backslash\{0\}, \alpha>0$, and $R>0$, we define a cone

$$
C(v, \alpha)=\left\{y \in \bar{M} \backslash\{x\}: \varangle\left(v, \dot{\gamma}_{0}^{x, y}\right)<\alpha\right\}
$$

and a truncated cone

$$
T(v, \alpha, R)=C(v, \alpha) \backslash \bar{B}(x, R)
$$

All cones and open balls in $M$ form a basis for the cone topology in $\bar{M}$. With this topology $\bar{M}$ is homeomorphic to the closed unit ball $\bar{B}^{n} \subset \mathbb{R}^{n}$ and $\partial_{\infty} M$ to the unit sphere $\mathbb{S}^{n-1}=\partial B^{n}$. For detailed study on the cone topology, see [16].

Let us recall that the local Sobolev inequality holds on any Cartan-Hadamard manifold $M$. More precisely, there exist constants $r_{S}>0$ and $C_{S}<\infty$ such that

$$
\left(\int_{B}|\eta|^{n /(n-1)}\right)^{(n-1) / n} \leq C_{S} \int_{B}|\nabla \eta|
$$

holds for every ball $B=B\left(x, r_{S}\right) \subset M$ and every function $\eta \in C_{0}^{\infty}(B)$. This inequality can be obtained e.g. from Croke's estimate of the isoperimetric constant, see [5] and [9].

2.2. Jacobi equation. If $k:[0, \infty) \rightarrow(-\infty, 0]$ is a smooth function, we denote by $f_{k} \in C^{\infty}([0, \infty))$ the solution to the initial value problem

$$
\left\{\begin{aligned}
f_{k}^{\prime \prime}+k f_{k} & =0 \\
f_{k}(0) & =0 \\
f_{k}^{\prime}(0) & =1 .
\end{aligned}\right.
$$

The solution is a non-negative smooth function.

In later sections we will need some known results related to Jacobi fields and curvature bounds. The proofs of the following three lemmas are based on the Rauch comparison theorem (see e.g. 18]) and can be found in 30. Concerning the curvature bounds, we have the following estimates for the growth of Jacobi fields and the Laplacian of the distance function:

Lemma 2.1. [30, Lemma 1] Let $k, K:[0, \infty) \rightarrow(-\infty, 0]$ be smooth functions that are constant in some neighborhood of 0 . Suppose that $v \in T_{o} M$ is a unit vector and $\gamma=\gamma^{v}: \mathbb{R} \rightarrow M$ is the unit speed geodesic with $\dot{\gamma}_{0}=v$. Suppose that for every $t>0$ we have

$$
k(t) \leq K_{M}(P) \leq K(t)
$$

for every two-dimensional subspace $P \subset T_{\gamma(t)} M$ that contains the radial vector $\dot{\gamma}_{t}$.

(1) If $W$ is a Jacobi field along $\gamma$ with $W_{0}=0,\left|W_{0}^{\prime}\right|=1$, and $W_{0}^{\prime} \perp v$, then

$$
f_{K}(t) \leq|W(t)| \leq f_{k}(t)
$$

for every $t \geq 0$.

(2) For every $t>0$ we have

$$
(n-1) \frac{f_{K}^{\prime}(t)}{f_{K}(t)} \leq \Delta r(\gamma(t)) \leq(n-1) \frac{f_{k}^{\prime}(t)}{f_{k}(t)} .
$$

The pinching condition for the sectional curvatures gives a relation between the maximal and minimal moduli of Jacobi fields along a given geodesic that contains the radial vector: 
Lemma 2.2. 6, Lemma 3.2][30, Lemma 3] Let $v \in T_{o} M$ be a unit vector and $\gamma=\gamma^{v}$. Suppose that $r_{0}>0$ and $k<0$ are constants such that $K_{M}(P) \geq k$ for every two-dimensional subspace $P \subset T_{x} M, x \in B\left(o, r_{0}\right)$. Suppose that there exists a constant $C_{K}<\infty$ such that

$$
\left|K_{M}(P)\right| \leq C_{K}\left|K_{M}\left(P^{\prime}\right)\right|
$$

whenever $t \geq r_{0}$ and $P, P^{\prime} \subset T_{\gamma(t)} M$ are two-dimensional subspaces containing the radial vector $\dot{\gamma}_{t}$. Let $V$ and $\bar{V}$ be two Jacobi fields along $\gamma$ such that $V_{0}=0=\bar{V}_{0}$, $V_{0}^{\prime} \perp \dot{\gamma}_{0} \perp \bar{V}_{0}$, and $\left|V_{0}^{\prime}\right|=1=\left|\bar{V}_{0}^{\prime}\right|$. Then there exists a constant $c_{0}=c_{0}\left(C_{K}, r_{0}, k\right)>0$ such that

for every $r \geq r_{0}$.

$$
\left|V_{r}\right|^{C_{K}} \geq c_{0}\left|\bar{V}_{r}\right|
$$

To prove the solvability of the minimal graph equation, we will need an estimate for the gradient of a certain angular function. This estimate can be obtained in terms of Jacobi fields:

Lemma 2.3. 30, Lemma 2] Let $x_{0} \in M \backslash\{o\}, U=M \backslash \gamma^{o, x_{0}}(\mathbb{R})$, and define $\theta: U \rightarrow[0, \pi], \theta(x)=\varangle_{o}\left(x_{0}, x\right):=\arccos \left\langle\dot{\gamma}_{0}^{o, x_{0}}, \dot{\gamma}_{0}^{o, x}\right\rangle$. Let $x \in U$ and $\gamma=\gamma^{o, x}$. Then there exists a Jacobi field $W$ along $\gamma$ with $W(0)=0, W_{0}^{\prime} \perp \dot{\gamma}_{0}$, and $\left|W_{0}^{\prime}\right|=1$ such that

$$
|\nabla \theta(x)| \leq \frac{1}{|W(r(x))|} .
$$

2.3. Young functions. Let $\phi:[0, \infty) \rightarrow[0, \infty)$ be a homeomorphism and let $\psi=$ $\phi^{-1}$. Define Young functions $\Phi$ and $\Psi$ by setting

$$
\Phi(t)=\int_{0}^{t} \phi(s) d s
$$

and

$$
\Psi(t)=\int_{0}^{t} \psi(s) d s
$$

for each $t \in[0, \infty)$. Then we have the following Young's inequality

$$
a b \leq \Phi(a)+\Psi(b)
$$

for all $a, b \in[0, \infty)$. The functions $\Phi$ and $\Psi$ are said to form a complementary Young pair. Furthermore, $\Phi$ (and similarly $\Psi$ ) is a continuous, strictly increasing, and convex function satisfying

$$
\lim _{t \rightarrow 0^{+}} \frac{\Phi(t)}{t}=0
$$

and

$$
\lim _{t \rightarrow \infty} \frac{\Phi(t)}{t}=\infty
$$

For a more general definition of Young functions see e.g. [24].

As in 31, we consider complementary Young pairs of a special type. For that, suppose that a homeomorphism $G:[0, \infty) \rightarrow[0, \infty)$ is a Young function that is a diffeomorphism on $(0, \infty)$ and satisfies

$$
\int_{0}^{1} \frac{d t}{G^{-1}(t)}<\infty
$$

and

$$
\lim _{t \rightarrow 0} \frac{t G^{\prime}(t)}{G(t)}=1 .
$$

Then we define $F:[0, \infty) \rightarrow[0, \infty)$ so that $G$ and $F$ form a complementary Young pair. The space of such functions $F$ will be denoted by $\mathcal{F}$. Note that if $F \in \mathcal{F}$, 
then also $\lambda F \in \mathcal{F}$ and $F(\lambda \cdot) \in \mathcal{F}$ for every $\lambda>0$. In 31] it is proved that for fixed $\varepsilon_{0} \in(0,1)$ there exists $F \in \mathcal{F}$ such that

$$
F(t) \leq t^{1+\varepsilon_{0}} \exp \left(-\frac{1}{t}\left(\log \left(e+\frac{1}{t}\right)\right)^{-1-\varepsilon_{0}}\right)
$$

for all $t \in[0, \infty)$. The construction of such $F$ is done by first choosing $\lambda \in\left(1,1+\varepsilon_{0}\right)$ and a homeomorphism $H:[0, \infty) \rightarrow[0, \infty)$ that is a diffeomorphism on $(0, \infty)$ and satisfies

$$
H(t)= \begin{cases}\left(\log \frac{1}{t}\right)^{-1}\left(\log \log \frac{1}{t}\right)^{-\lambda} & \text { if } t \text { is small enough, } \\ t^{1 / \varepsilon_{0}} & \text { if } t \text { is large enough, }\end{cases}
$$

and then setting $G(t)=\int_{0}^{t} H(s) d s$ and $F(t)=\int_{0}^{t} H^{-1}(s) d s$. From now on, $G$ and $F$ will denote the complementary Young pair obtained via this procedure. For details, see 31 and the proof of Proposition 2.5 below.

Since $G$ is convex, we have $G(t) \geq c t$ for all $t \geq 1$. Therefore $G^{-1}(t) \leq c t$ for all $t$ large enough and this implies that $\int_{0}^{\infty} 1 / G^{-1}=\infty$. From this, together with 2.3 , we conclude that the function $\psi$, defined by

$$
\psi(t)=\int_{0}^{t} \frac{d s}{G^{-1}(s)}
$$

is a homeomorphism $[0, \infty) \rightarrow[0, \infty)$ that is a diffeomorphism on $(0, \infty)$. Hence the same is true for its inverse

$$
\varphi=\psi^{-1}:[0, \infty) \rightarrow[0, \infty) .
$$

The following lemma collects the properties of $\varphi$.

Lemma 2.4. 31, Lemma 4.5] The function $\varphi:[0, \infty) \rightarrow[0, \infty)$ is a homeomorphism that is smooth on $(0, \infty)$ and satisfies

$$
G \circ \varphi^{\prime}=\varphi
$$

and

$$
\lim _{t \rightarrow 0+} \frac{\varphi^{\prime \prime}(t) \varphi(t)}{\varphi^{\prime}(t)^{2}}=1
$$

From now on, $\varphi$ will be the function defined in 2.7 such that the corresponding $F \in \mathcal{F}$ satisfies 2.5 . Using the computations done in 31, we obtain a more specific formula for the function $\varphi$. Namely, we know that $G^{-1}(t) \approx t / H(t)$ and hence

$$
\psi(t)=\int_{0}^{t} \frac{d s}{G^{-1}(s)} \approx \int_{0}^{t} \frac{1}{s\left(\log \frac{1}{s}\right)\left(\log \log \frac{1}{s}\right)^{1+\varepsilon_{0}}}=\frac{1}{\varepsilon_{0}}\left(\log \log \frac{1}{t}\right)^{-\varepsilon_{0}} .
$$

Here and in what follows $\approx$ means that the ratio of the two sides tends to 1 as $t \rightarrow 0^{+}$. From this it is straightforward to see that

$$
\varphi(t) \approx \exp \left(-\exp \left(\frac{1}{\varepsilon_{0} t}\right)^{\varepsilon_{0}}\right)
$$

We will also need complementary Young functions $G_{1}$ and $F_{1}$ to deal with the second derivative of the function $\varphi$. The existence of these functions will be proved by the following proposition which is just a modification of [31, Proposition 4.3] since in the construction of the Young functions we will replace the function $H$ in 31 by $H^{2}$.

Proposition 2.5. Let $\varepsilon_{0} \in(0,1)$ and $\lambda \in\left(1,1+\varepsilon_{0}\right)$ be as in $(2.6)$. Then there exist complementary Young functions $G_{1}$ and $F_{1}$, and a constant $c>0$ such that $G_{1}$ satisfies

$$
G_{1}\left(\varphi^{\prime \prime}(t)\right) \approx \varphi(t)
$$


and $F_{1}$ satisfies

$$
F_{1}(t) \leq c t \exp \left(-\frac{2^{\lambda}}{\sqrt{t}}\left(\log \frac{1}{t}\right)^{-\lambda}\right)
$$

for all sufficiently small $t>0$.

Proof. Let $H:[0, \infty) \rightarrow[0, \infty)$ be as in 2.6 . We define $G_{1}(t)=\int_{0}^{t} H(s)^{2} d s$. Then $G_{1}$ is a Young function and we denote by $F_{1}$ its Young conjugate. Notice that $G_{1}^{\prime}(t)=H(t)^{2}$ and that $t\left(H^{2}\right)^{\prime}(t) / H(t)^{2} \rightarrow 0$ as $t \rightarrow 0$. Hence, by l'Hospital's rule, we have

$$
\lim _{t \rightarrow 0} \frac{t G_{1}^{\prime}(t)}{G_{1}(t)}=\lim _{t \rightarrow 0} \frac{\frac{d}{d t}\left(t G_{1}^{\prime}(t)\right)}{G_{1}^{\prime}(t)}=1
$$

and we see that $G_{1}$ satisfies 2.4. Next, denote $R(t)=t / H(t)^{2}$. Then it is easy to see that $R(k t) \approx k R(t)$ for every constant $k>0$ and we get

$$
R\left(G_{1}(t)\right) \approx R\left(t H(t)^{2}\right)=\frac{t H(t)^{2}}{H\left(t H(t)^{2}\right)^{2}} \approx t,
$$

which gives us $G_{1}^{-1}(t) \approx R(t)$. It follows that $G_{1}$ satisfies 2.3 and hence $F_{1} \in \mathcal{F}$. On the other hand $\varphi(t)=\psi^{-1}(t)$ and

$$
\psi^{\prime}(t)=\frac{1}{G^{-1}(t)} \approx \frac{H(t)}{t},
$$

and therefore

By (2.9) we obtain

$$
\varphi^{\prime}(t)=\frac{1}{\psi^{\prime}(\varphi(t))} \approx \frac{\varphi(t)}{H(\varphi(t))} .
$$

$$
\varphi^{\prime \prime}(t) \approx \frac{\varphi(t)}{H(\varphi(t))^{2}}=R(\varphi(t)) \approx G_{1}^{-1}(\varphi(t)),
$$

and so

$$
G_{1}\left(\varphi^{\prime \prime}(t)\right) \approx \varphi(t) .
$$

Thus we are left to estimate $F_{1}$ from above.

It is straightforward to check that

$$
\left(H^{2}\right)^{-1}(t)=\exp \left(-\exp \left(\lambda W\left(\lambda^{-1} t^{-1 /(2 \lambda)}\right)\right)\right)
$$

for all sufficiently small $t$, where $W$ is the Lambert $W$ function defined by the identity $W(s) e^{W(s)}=s$. Since $F_{1}^{\prime}(t)=\left(G_{1}^{\prime}\right)^{-1}(t)=\left(H^{2}\right)^{-1}(t)$ and $W(s) \geq \log s-$ $\log \log s$ for all $s \geq e$, we get for sufficiently small $t$

$$
\begin{aligned}
F_{1}(t) & =\int_{0}^{t}\left(H^{2}\right)^{-1}(s) d s \leq t\left(H^{2}\right)^{-1}(t) \\
& =\frac{t}{\exp \left(\exp \left(\lambda W\left(\lambda^{-1} t^{-1 / 2 \lambda}\right)\right)\right)} \\
& \leq \frac{t}{\exp \left(\exp \left(\lambda \log \left(\lambda^{-1} t^{-1 / 2 \lambda}\right)-\lambda \log \log \left(\lambda^{-1} t^{-1 / 2 \lambda}\right)\right)\right)} \\
& =\frac{t}{\exp \left(\left(\lambda^{-1} t^{-1 / 2 \lambda}\right)^{\lambda}\left(\log \left(\lambda^{-1} t^{-1 / 2 \lambda}\right)\right)^{\lambda}\right)} \\
& =t \exp \left(-\frac{1}{\lambda^{\lambda} \sqrt{t}}\left(\log \frac{1}{\lambda}+\frac{1}{2 \lambda} \log \frac{1}{t}\right)^{-\lambda}\right) \\
& \leq c t \exp \left(-\frac{2^{\lambda}}{\sqrt{t}}\left(\log \frac{1}{t}\right)^{-\lambda}\right)
\end{aligned}
$$


2.4. Minimal graph equation. Let $\Omega \subset M$ be an open set. Then a function $u \in W_{\mathrm{loc}}^{1,1}(\Omega)$ is a (weak) solution of the minimal graph equation if

$$
\int_{\Omega} \frac{\langle\nabla u, \nabla \varphi\rangle}{\sqrt{1+|\nabla u|^{2}}}=0
$$

for every $\varphi \in C_{0}^{\infty}(\Omega)$. Note that the integral is well-defined since

$$
\sqrt{1+|\nabla u|^{2}} \geq|\nabla u| \text { a.e. }
$$

and thus

$$
\int_{\Omega} \frac{|\langle\nabla u, \nabla \varphi\rangle|}{\sqrt{1+|\nabla u|^{2}}} \leq \int_{\Omega} \frac{|\nabla u||\nabla \varphi|}{\sqrt{1+|\nabla u|^{2}}} \leq \int_{\Omega}|\nabla \varphi|<\infty .
$$

It is known that under certain conditions there exists a (strong) solution of (1.1) with given boundary values. Namely, let $\Omega \subset \subset M$ be a smooth relatively compact open set whose boundary has positive mean curvature with respect to inwards pointing unit normal. Then for each $f \in C^{2, \alpha}(\bar{\Omega})$ there exists a unique $u \in C^{\infty}(\Omega) \cap C^{2, \alpha}(\bar{\Omega})$ that solves the minimal graph equation $(1.1)$ in $\Omega$ and has the boundary values $u|\partial \Omega=f| \partial \Omega$.

\section{Asymptotic Dirichlet problem for minimal GRAPh EQUATion}

We begin by the following Caccioppoli-type inequality which will have a crucial role in the proof of the solvability of the minimal graph equation.

Lemma 3.1. Suppose $\varphi:[0, \infty) \rightarrow[0, \infty)$ is a homeomorphism that is smooth on $(0, \infty)$ and let $U \subset \subset M$ be open. Suppose that $\eta \geq 0$ is a $C^{1}(U)$ function and let $u, \theta \in L^{\infty}(U) \cap W^{1,2}(U)$ be continuous functions such that $u \in C^{2}(U)$ is a solution to the minimal graph equation (1.1) in $U$. Denote

$$
h=\frac{|u-\theta|}{\nu},
$$

where $\nu>0$ is a constant, and assume that

$$
\eta^{2} \varphi(h) \in W_{0}^{1,2}(U)
$$

Then we have

$$
\int_{U} \eta^{2} \varphi^{\prime}(h) \frac{|\nabla u|^{2}}{\sqrt{1+|\nabla u|^{2}}} \leq C_{\varepsilon} \int_{U} \eta^{2} \varphi^{\prime}(h)|\nabla \theta|^{2}+(4+\varepsilon) \nu^{2} \int_{U} \frac{\varphi^{2}}{\varphi^{\prime}}(h)|\nabla \eta|^{2}
$$

for any fixed $\varepsilon>0$.

Proof. Define an auxiliary function $f$ by

$$
f=\eta^{2} \varphi\left(\frac{(u-\theta)^{+}}{\nu}\right)-\eta^{2} \varphi\left(\frac{(u-\theta)^{-}}{\nu}\right) .
$$

Then it holds that $f \in W_{0}^{1,2}(U)$ and its gradient is given by

$$
\nabla f=\frac{1}{\nu} \eta^{2} \varphi^{\prime}(h)(\nabla u-\nabla \theta)+2 \eta \operatorname{sgn}(u-\theta) \varphi(h) \nabla \eta .
$$

Since $u$ is a solution to the minimal graph equation, we can use $f$ as a test function in

$$
\int_{U} \frac{\langle\nabla u, \nabla f\rangle}{\sqrt{1+|\nabla u|^{2}}}=0
$$


and obtain

$$
\begin{aligned}
\int_{U} \eta^{2} \varphi^{\prime}(h) \frac{|\nabla u|^{2}}{\sqrt{1+|\nabla u|^{2}}}= & \int_{U} \eta^{2} \varphi^{\prime}(h) \frac{\langle\nabla u, \nabla \theta\rangle}{\sqrt{1+|\nabla u|^{2}}} \\
& -2 \nu \int_{U} \eta \operatorname{sgn}(u-\theta) \varphi(h) \frac{\langle\nabla u, \nabla \eta\rangle}{\sqrt{1+|\nabla u|^{2}}} \\
\leq & \int_{U} \eta^{2} \varphi^{\prime}(h) \frac{|\nabla u||\nabla \theta|}{\sqrt{1+|\nabla u|^{2}}}+2 \nu \int_{U} \eta \varphi(h) \frac{|\nabla u||\nabla \eta|}{\sqrt{1+|\nabla u|^{2}}} .
\end{aligned}
$$

Next we use Young's inequality $a b \leq(\varepsilon / 2) a^{2}+1 /(2 \varepsilon) b^{2}$ and $\sqrt{1+|\nabla u|^{2}} \geq 1$ to estimate the terms on the right hand side as

$$
\int_{U} \eta^{2} \varphi^{\prime}(h) \frac{|\nabla u||\nabla \theta|}{\sqrt{1+|\nabla u|^{2}}} \leq \frac{\varepsilon_{1}}{2} \int_{U} \eta^{2} \varphi^{\prime}(h) \frac{|\nabla u|^{2}}{\sqrt{1+|\nabla u|^{2}}}+\frac{1}{2 \varepsilon_{1}} \int_{U} \eta^{2} \varphi^{\prime}(h)|\nabla \theta|^{2}
$$

and

$$
2 \nu \int_{U} \eta \varphi(h) \frac{|\nabla u||\nabla \eta|}{\sqrt{1+|\nabla u|^{2}}} \leq \varepsilon_{2} \int_{U} \eta^{2} \varphi^{\prime}(h) \frac{|\nabla u|^{2}}{\sqrt{1+|\nabla u|^{2}}}+\frac{\nu^{2}}{\varepsilon_{2}} \int_{U} \frac{\varphi^{2}}{\varphi^{\prime}}(h)|\nabla \eta|^{2} .
$$

Then we choose $\varepsilon_{1}$ and $\varepsilon_{2}$ such that $\varepsilon_{1}$ is small enough and $\varepsilon_{2}$ minimizes the term

$$
\frac{1}{\varepsilon_{2}\left(1-\varepsilon_{1} / 2-\varepsilon_{2}\right)}
$$

i.e. $\varepsilon_{2}=\left(2-\varepsilon_{1}\right) / 4$. Combining all terms we arrive at

$$
\begin{aligned}
\int_{U} \eta^{2} \varphi^{\prime}(h) \frac{|\nabla u|^{2}}{\sqrt{1+|\nabla u|^{2}}} & \leq \frac{2}{\varepsilon_{1}\left(2-\varepsilon_{1}\right)} \int_{U} \eta^{2} \varphi^{\prime}(h)|\nabla \theta|^{2}+\frac{4 \nu^{2}}{1-\varepsilon_{1}} \int_{U} \frac{\varphi^{2}}{\varphi^{\prime}}(h)|\nabla \eta|^{2} \\
& =C_{\varepsilon} \int_{U} \eta^{2} \varphi^{\prime}(h)|\nabla \theta|^{2}+(4+\varepsilon) \nu^{2} \int_{U} \frac{\varphi^{2}}{\varphi^{\prime}}(h)|\nabla \eta|^{2} .
\end{aligned}
$$

Remark 3.2. As can be seen in the proof of Lemma 3.3, the second term

$$
(4+\varepsilon) \nu^{2} \int_{U} \frac{\varphi^{2}}{\varphi^{\prime}}(h)|\nabla \eta|^{2}
$$

on the right hand side of (3.1) is the only term that affects to the dimensioncurvature restriction.

We notice that the left hand side of 3.1 can be estimated from below by

$$
\int_{U} \eta^{2} \varphi^{\prime}(h) \frac{|\nabla u|^{2}}{\sqrt{1+|\nabla u|^{2}}} \geq c_{1} \int_{U_{1}} \eta^{2} \varphi^{\prime}(h)|\nabla u|^{2}+c_{2} \int_{U_{2}} \eta^{2} \varphi^{\prime}(h)|\nabla u|
$$

where

$$
U_{1}=\{|\nabla u| \leq \sigma\}, \quad U_{2}=\{|\nabla u| \geq \sigma\}, \quad \sigma>0
$$

and

$$
c_{1}=\frac{1}{\sqrt{1+\sigma^{2}}}, \quad c_{2}=\frac{1}{\sqrt{1+\left(1 / \sigma^{2}\right)}} .
$$

In the following Lemmas we will obtain some estimates using Lipschitz data $\theta: M \rightarrow \mathbb{R}$. By Rademacher's theorem, Lipschitz functions are differentiable almost everywhere and throughout the computations, the gradient $\nabla \theta$ appears only inside integrals so the points where $\theta$ is not differentiable will not be a problem.

Before stating the Lemmas we introduce the following notation. For $x \in M$, we denote by $j(x)$ the infimum of $|V(r(x))|$ over Jacobi fields $\mathrm{V}$ along the geodesic 
$\gamma^{o, x}$ that satisfy $V_{0}=0,\left|V_{0}^{\prime}\right|=1$ and $V_{0}^{\prime} \perp \dot{\gamma}_{0}^{o, x}$. We also note that since $M$ is a Cartan-Hadamard manifold, we have

$$
\Delta r \geq \frac{n-1}{r}
$$

in $M \backslash\{o\}$. From the curvature upper bound, Lemma 2.1 and [30, Example 1] it follows that for every $\varepsilon>0$ there exists $R_{1}>R_{0}$ such that

$$
\Delta r \geq \frac{(n-1) \phi}{(1+\varepsilon) r}
$$

for $r \geq R_{1}$ and therefore

$$
r \Delta r \geq \begin{cases}n-1, & \text { in } M \backslash\{o\}, \\ \frac{(n-1) \phi}{1+\varepsilon}, & \text { in } M \backslash B\left(o, R_{1}\right) .\end{cases}
$$

Lemma 3.3. Let $M$ be a Cartan-Hadamard manifold satisfying

$$
K(P) \leq-\frac{\phi(\phi-1)}{r(x)^{2}}
$$

where $K(P)$ is the sectional curvature of any plane $P \subset T_{x} M$ that contains the radial vector field $\nabla r(x)$ and $x$ is any point in $M \backslash B\left(o, R_{0}\right)$. Furthemore, suppose that the dimension of $M$ and the constant $\phi$ satisfies the relation $(1.6)$. Let $U=$ $B(o, R)$, with $R>R_{1}$, and suppose that $u \in C^{2}(U) \cap C(\bar{U})$ is the unique solution to the minimal graph equation in $U$, with $u|\partial U=\theta| \partial U$, where $\theta: M \rightarrow \mathbb{R}$ is a Lipschitz function, with $|\nabla \theta(x)| \leq 1 / j(x)$ almost everywhere. Then there exists a constant $c$ independent of $u$ such that

$$
\int_{U} \varphi(|u-\theta| / c) \leq c+c \int_{U} F(r|\nabla \theta|)+c \int_{U} F_{1}\left(r^{2}|\nabla \theta|^{2}\right) .
$$

Proof. As before, we denote $h=|u-\theta| / \nu$, where $\nu \geq \nu_{0}$ will be fixed later, and to shorten the notation we denote $(n-1) \phi /(1+\varepsilon)=: C_{0}$. By splitting the integration domain and using the estimate 3.3$)$, we first obtain

$$
\begin{aligned}
\int_{U} \varphi(h) r \Delta r & =\int_{B\left(o, R_{1}\right)} \varphi(h) r \Delta r+\int_{U \backslash B\left(o, R_{1}\right)} \varphi(h) r \Delta r \\
& \geq(n-1) \int_{B\left(o, R_{1}\right)} \varphi(h)+C_{0} \int_{U \backslash B\left(o, R_{1}\right)} \varphi(h) \\
& \geq\left(n-1-C_{0}\right) \int_{B\left(o, R_{1}\right)} \varphi(h)+C_{0} \int_{U} \varphi(h) \\
& \geq-c+C_{0} \int_{U} \varphi(h),
\end{aligned}
$$

where $c \geq 0$ is some constant. Next we use Green's formula to obtain

$$
\begin{aligned}
-c+C_{0} \int_{U} \varphi(h) \leq \int_{U} \varphi(h) r \Delta r & =-\int_{U}\langle\nabla(\varphi(h) r), \nabla r\rangle \\
& =-\int_{U} \varphi(h)-\int_{U} r \varphi^{\prime}(h)\langle\nabla h, \nabla r\rangle,
\end{aligned}
$$

and consequently we have

$$
-c+\left(1+C_{0}\right) \int_{U} \varphi(h) \leq \int_{U} r \varphi^{\prime}(h)|\nabla h| .
$$

To estimate the right hand side term, we first split the integration domain into two pieces $U=U_{1} \cup U_{2}$, where

$$
U_{1}=\{x \in U:|\nabla u| \leq \sigma\} \quad \text { and } \quad U_{2}=\{x \in U:|\nabla u|>\sigma\} .
$$


Note that $|\nabla h| \leq|\nabla u| / \nu+|\nabla \theta| / \nu$, so using the Caccioppoli-type inequality 3.1 and $(3.2)$ we get

$$
\begin{aligned}
\int_{U} r \varphi^{\prime}(h)|\nabla h| \leq & \frac{1}{\nu} \int_{U_{1}} r \varphi^{\prime}(h)|\nabla u|+\frac{1}{\nu} \int_{U_{2}} r \varphi^{\prime}(h)|\nabla u|+\frac{1}{\nu} \int_{U} r \varphi^{\prime}(h)|\nabla \theta| \\
\leq & \frac{1}{\nu} \int_{U_{1}} r \varphi^{\prime}(h)|\nabla u|+\frac{1}{\nu} \int_{U} r \varphi^{\prime}(h)|\nabla \theta| \\
& \quad+\frac{C_{\varepsilon}}{c_{2} \nu} \int_{U} r \varphi^{\prime}(h)|\nabla \theta|^{2}+\frac{(4+\varepsilon) \nu}{c_{2}} \int_{U} \frac{\varphi^{2}}{\varphi^{\prime}}(h)|\nabla \sqrt{r}|^{2} \\
= & \frac{1}{\nu} \int_{U_{1}} r \varphi^{\prime}(h)|\nabla u|+\frac{1}{\nu} \int_{U} r \varphi^{\prime}(h)|\nabla \theta| \\
& \quad+\frac{C_{\varepsilon}}{c_{2} \nu} \int_{U} r \varphi^{\prime}(h)|\nabla \theta|^{2}+\frac{(4+\varepsilon) \nu}{4 c_{2}} \int_{U} \frac{\varphi^{2}}{\varphi^{\prime}}(h) r^{-1}
\end{aligned}
$$

By 2.8 and the convexity of the Young function $G$ we have $\varphi(h) \leq c \varphi^{\prime}(h)$, and for $r$ large enough, $|\nabla \theta|<1$, so $|\nabla \theta|^{2} \leq|\nabla \theta|$. So from the previous estimate, we deduce that

$$
\int_{U} r \varphi^{\prime}(h)|\nabla h| \leq \frac{1}{\nu} \int_{U_{1}} r \varphi^{\prime}(h)|\nabla u|+\frac{1+C_{\varepsilon} / c_{2}}{\nu} \int_{U} r \varphi^{\prime}(h)|\nabla \theta|+c+\varepsilon^{\prime} \int_{U} \varphi(h) .
$$

We continue again by splitting $U_{1}$ into two pieces by $U_{1}=U_{3} \cup U_{4}$, where

$$
U_{3}=\left\{|\nabla u| \leq \tilde{\sigma} \frac{\varphi(h)}{\varphi^{\prime}(h) r}\right\} \quad \text { and } \quad U_{4}=\left\{\tilde{\sigma} \frac{\varphi(h)}{\varphi^{\prime}(h) r}<|\nabla u| \leq \sigma\right\}
$$

and $\tilde{\sigma}$ is a constant to be determined later. Denote $\Psi(t):=\int_{0}^{t} \varphi^{\prime}(s)^{2} / \varphi(s) d s$. Then using the Caccioppoli-type inequality (3.1) and 3.2 with $r$ and $\Psi^{\prime}$ instead of $\eta$ and $\varphi^{\prime}$ respectively, we can estimate the integral over $U_{1}$ by

$$
\begin{aligned}
\int_{U_{1}} r \varphi^{\prime}(h)|\nabla u| \leq \tilde{\sigma} \int_{U_{3}} \varphi(h)+\frac{1}{\tilde{\sigma}} \int_{U_{4}} r^{2} \frac{\varphi^{\prime}(h)^{2}}{\varphi(h)}|\nabla u|^{2} \\
\quad \leq \tilde{\sigma} \int_{U_{3}} \varphi(h)+\frac{1}{\tilde{\sigma}}\left(\frac{C_{\varepsilon}}{c_{1}} \int_{U} r^{2} \Psi^{\prime}(h)|\nabla \theta|^{2}+\frac{(4+\varepsilon) \nu^{2}}{c_{1}} \int_{U} \frac{\Psi^{2}}{\Psi^{\prime}}(h)\right) .
\end{aligned}
$$

From 2.9 we see that

$$
\Psi^{\prime}(t)=\frac{\varphi^{\prime}(t)^{2}}{\varphi(t)} \leq \tilde{c} \varphi^{\prime \prime}(t)
$$

for $t$ small enough, and hence

$$
\Psi(t)=\int_{0}^{t} \frac{\varphi^{\prime}(s)^{2}}{\varphi(s)} \leq \tilde{c} \varphi^{\prime}(t)
$$

which implies that

$$
\frac{\Psi^{2}}{\Psi^{\prime}}(h) \leq \tilde{c} \frac{\varphi^{\prime}(h)^{2}}{\varphi^{\prime}(h)^{2} / \varphi(h)}=\tilde{c} \varphi(h) .
$$

Notice that $\tilde{c}$, as well as $c_{1}$, can be chosen arbitrarly close to 1 . Collecting these estimates together we arrive at

$$
\begin{aligned}
\left(1+C_{0}\right) \int_{U} \varphi(h) \leq c & +\varepsilon^{\prime} \int_{U} \varphi(h)+\frac{\tilde{\sigma}}{\nu} \int_{U} \varphi(h)+\frac{1+C_{\varepsilon} / c_{2}}{\nu} \int_{U} r \varphi^{\prime}(h)|\nabla \theta| \\
& +\frac{C_{\varepsilon} \tilde{c}}{c_{1} \tilde{\sigma} \nu} \int_{U} r^{2} \varphi^{\prime \prime}(h)|\nabla \theta|^{2}+\frac{(4+\varepsilon) \nu \tilde{c}}{c_{1} \tilde{\sigma}} \int_{U} \varphi(h) .
\end{aligned}
$$


Next we use the complementary Young functions $G$ and $F$ to estimate the term with $\varphi^{\prime}$, and $G_{1}$ and $F_{1}$ to estimate the term with $\varphi^{\prime \prime}$. So all together we have

$$
\begin{aligned}
\left(1+C_{0}-\varepsilon^{\prime}-\frac{1+C_{\varepsilon} / c_{2}}{\nu}\right. & \left.-\frac{\tilde{\sigma}}{\nu}-\frac{C_{\varepsilon} \tilde{c}}{c_{1} \tilde{\sigma} \nu}-\frac{(4+\varepsilon) \nu \tilde{c}}{c_{1} \tilde{\sigma}}\right) \int_{U} \varphi(h) \\
& \leq c+\frac{1+C_{\varepsilon} / c_{2}}{\nu} \int_{U} F(r|\nabla \theta|)+\frac{C_{\varepsilon}}{c_{1} \tilde{\sigma} \nu} \int_{U} F_{1}\left(r^{2}|\nabla \theta|^{2}\right) .
\end{aligned}
$$

For any fixed $\tilde{\varepsilon}>0$, we can choose first $\sigma$ and $\varepsilon$ small enough, then $\nu$ big enough and $\tilde{\sigma}=\nu$ such that the coefficient on the left hand side is positive provided that $C_{0}>4+\tilde{\varepsilon}$. This last inequality is satisfied thanks to the dimension-curvature restriction $(1.6)$ and hence the claim is proved.

The next lemma is a modification of [4, Lemma 20] (or originally [31, Lemma $2.20])$. The proof is based on the idea of Moser iteration procedure.

Lemma 3.4. Let $\Omega=B(o, R)$ and suppose that $\theta: \Omega \rightarrow \mathbb{R}$ is a bounded Lipschitz function with $|\theta|,|\nabla \theta| \leq C_{1}$. Let $u \in C^{2}(\Omega)$ be a solution of the minimal graph equation in $\Omega$ such that $u$ has the boundary values $\theta$ and $\inf _{\Omega} \theta \leq u \leq \sup _{\Omega} \theta$. Fix $s \in\left(0, r_{S}\right)$, where $r_{S}$ is the radius of the Sobolev inequality (2.1), and suppose that $B=B(x, s) \subset \Omega$. Then there exists a positive constant $\nu_{0}=\nu_{0}\left(\varphi, C_{1}\right)$ such that for all fixed $\nu \geq \nu_{0}$

$$
\sup _{B(x, s / 2)} \varphi(|u-\theta| / \nu)^{n+1} \leq c \int_{B} \varphi(|u-\theta| / \nu),
$$

where $c$ is a positive constant depending only on $n, \nu, s, C_{S}, C_{1}$ and $\varphi$.

Remark 3.5. Before proving the Lemma we note that increasing the constant $\nu$ above increases also the constant $c$. However, it does not cause problems since $\nu$ will always be a fixed constant.

Proof of Lemma 3.4. We denote $\kappa=n /(n-1), B / 2=B(x, s / 2)$, and $h=|u-\theta| / \nu$, where $\nu \geq \nu_{0}>0$ will be fixed later. For each $j \in \mathbb{N}$ we denote $s_{j}=s\left(1+\kappa^{-j}\right) / 2$ and $B_{j}=B\left(x, s_{j}\right)$. Note that $s_{j} \rightarrow s / 2$ as $j \rightarrow \infty$. Let $\eta_{j}$ be a Lipschitz function such that $0 \leq \eta_{j} \leq 1, \eta_{j}\left|B_{j+1} \equiv 1, \eta_{j}\right|\left(M \backslash B_{j}\right) \equiv 0$, and that

$$
\left|\nabla \eta_{j}\right| \leq \frac{1}{s_{j}-s_{j+1}}=2 n \kappa^{j} / s
$$

For every $m \geq 1$, we have

$$
\left|\nabla \eta_{j}^{2} \varphi(h)^{m}\right| \leq 2 \eta_{j} \varphi(h)^{m}\left|\nabla \eta_{j}\right|+m \eta_{j}^{2} \varphi^{\prime}(h) \varphi^{m-1}(h)|\nabla h| .
$$

First we claim that

$$
\left(\int_{B_{j+1}} \varphi(h)^{\kappa m}\right)^{1 / \kappa} \leq c\left(\kappa^{j}+m+\kappa^{2 j} / m\right) \int_{B_{j}} \varphi^{m-1} .
$$


We notice that, for every $m, j \geq 1, \eta_{j}^{2} \varphi(h)^{m}$ is a Lipschitz function supported in $B_{j}$. Using the Sobolev inequality 2.1 , we first have

$$
\begin{aligned}
& \left(\int_{B_{j+1}} \varphi(h)^{\kappa m}\right)^{1 / \kappa} \leq\left(\int_{B_{j}}\left(\eta_{j}^{2} \varphi(h)^{m}\right)^{\kappa}\right)^{1 / \kappa} \leq C_{S} \int_{B_{j}}\left|\nabla\left(\eta_{j} \varphi(h)^{m}\right)\right| \\
& \leq 2 C_{S} \int_{B_{j}} \eta_{j} \varphi(h)^{m}\left|\nabla \eta_{j}\right|+C_{S} \int_{B_{j}} \eta_{j}^{2}\left(\varphi^{m}\right)^{\prime}(h)|\nabla h| \\
& \leq c \kappa^{j} \int_{B_{j}} \varphi(h)^{m}+\frac{C_{S}}{\nu} \int_{B_{j}}\left(\varphi^{m}\right)^{\prime}(h)|\nabla \theta| \\
& \quad+\frac{C_{S}}{\nu} \int_{B_{j}} \eta_{j}^{2}\left(\varphi^{m}\right)^{\prime}(h)|\nabla u| .
\end{aligned}
$$

From the assumption

$$
-C_{1} \leq \inf _{\Omega} \theta \leq u \leq \sup _{\Omega} \theta \leq C_{1}
$$

we obtain that $|u-\theta| \leq 2 C_{1}$. We can use this to obtain upper bounds for $\varphi$ and $\varphi^{\prime}$. Namely, we have $G \circ \varphi^{\prime}=\varphi$, where $G:[0, \infty) \rightarrow[0, \infty)$ is the homeomorphic and convex Young function. Consequently there exist constants $\nu_{0}$ and $c$ such that

$$
\varphi(h) \leq 1, \varphi^{\prime}(h) \leq 1 \text { and } \varphi(h) \leq c \varphi^{\prime}(h)
$$

whenever $\nu \geq \nu_{0}$. Thus we get estimates

$$
\int_{B_{j}} \varphi(h)^{m} \leq \int_{B_{j}} \varphi(h)^{m-1}
$$

and

$$
\int_{B_{j}}\left(\varphi^{m}\right)^{\prime}(h)|\nabla \theta|=m \int_{B_{j}} \varphi(h)^{m-1} \varphi^{\prime}(h)|\nabla \theta| \leq m C_{1} \int_{B_{j}} \varphi(h)^{m-1} .
$$

The third term on the right hand side of 3.5 can be estimated first as

$$
\begin{aligned}
\int_{B_{j}} \eta_{j}^{2}\left(\varphi^{m}\right)^{\prime}(h)|\nabla u| & \leq \int_{B_{j} \cap U_{1}} \eta_{j}^{2}\left(\varphi^{m}\right)^{\prime}(h)+\int_{B_{j} \cap U_{2}} \eta_{j}^{2}\left(\varphi^{m}\right)^{\prime}(h)|\nabla u| \\
& \leq \int_{B_{j}} m \varphi(h)^{m-1}+\sqrt{2} \int_{B_{j}} \eta_{j}^{2}\left(\varphi^{m}\right)^{\prime}(h) \frac{|\nabla u|^{2}}{\sqrt{1+|\nabla u|^{2}}},
\end{aligned}
$$

where $U_{1}$ is the set where $|\nabla u|<1$ and $U_{2}$ the set where $|\nabla u| \geq 1$. The constant $\sqrt{2}$ comes from $(3.2$ when we choose $\sigma=1$.

Next we notice that $\eta_{j}^{2} \varphi(h)^{m} \in W_{0}^{1,2}\left(B_{j}\right)$, since supp $\eta_{j} \subset \bar{B}_{j}$, and thus we can apply the Caccioppoli-type inequality (3.1) with $\varphi^{m}$ instead of $\varphi$. We also choose $\varepsilon_{1}=\varepsilon_{2}=1 / 3$ in the proof of $(3.1)$ so the constants become 3 and 6 . Hence we obtain

$$
\begin{gathered}
\sqrt{2} \int_{B_{j}} \eta_{j}^{2}\left(\varphi^{m}\right)^{\prime}(h) \frac{|\nabla u|^{2}}{\sqrt{1+|\nabla u|^{2}}} \leq 3 \sqrt{2} \int_{B_{j}} \eta_{j}^{2}\left(\varphi^{m}\right)^{\prime}(h)|\nabla \theta|^{2} \\
+6 \sqrt{2} \nu^{2} \int_{B_{j}} \frac{\varphi^{2 m}}{\left(\varphi^{m}\right)^{\prime}}(h)\left|\nabla \eta_{j}\right|^{2} \\
\leq c\left(m+\kappa^{2 j} / m\right) \int_{B_{j}} \varphi(h)^{m-1} .
\end{gathered}
$$

Now the estimate (3.4) follows by inserting the estimates $(3.6)-(3.9)$ into $(3.5)$. We apply (3.4) with $m=m_{j}+1$, where $m_{j}=(n+1) \kappa^{j}-n$. Note that $m_{j+1}=\kappa\left(m_{j}+1\right)$, 
so we can write 3.4 as

$$
\left(\int_{B_{j+1}} \varphi(h)^{m_{j}}\right)^{1 / \kappa} \leq C \kappa^{j} \int_{B_{j}} \varphi(h)^{m_{j}} .
$$

By denoting

$$
I_{j}=\left(\int_{B_{j}} \varphi(h)^{m_{j}}\right)^{1 / \kappa^{j}}
$$

we can write the previous inequality as a recursion formula

$$
I_{j+1} \leq C^{1 / \kappa^{j}} \kappa^{j / \kappa^{j}} I_{j} .
$$

Since

$$
\limsup _{j \rightarrow \infty} I_{j} \geq \lim _{j \rightarrow \infty}\left(\int_{B / 2} \varphi(h)^{m_{j}}\right)^{(n+1) / m_{j}}=\sup _{B / 2} \varphi(h)^{n+1},
$$

we get

$$
\sup _{B / 2} \varphi(h)^{n+1} \leq \limsup _{j \rightarrow \infty} I_{j} \leq C^{n} \kappa^{S} I_{0} \leq c \int_{B} \varphi(h),
$$

where

$$
S=\sum_{j=0}^{\infty} j \kappa^{-j}<\infty
$$

In order to prove that our solution to the minimal graph equation extends to the boundary $\partial_{\infty} M$ and has the desired boundary values, we will also need that the right hand side integrals of Lemma 3.3 are finite. The following ensures that the functions $F$ and $F_{1}$ decrease fast enough. Recall that $j(x)$ denotes the infimum of $|V(r(x))|$ over Jacobi fields $\mathrm{V}$ along the geodesic $\gamma^{o, x}$ that satisfy $V_{0}=0,\left|V_{0}^{\prime}\right|=1$ and $V_{0}^{\prime} \perp \dot{\gamma}_{0}^{o, x}$.

Lemma 3.6. Let $M$ be a Cartan-Hadamard manifold satisfying

$$
K(P) \leq-\frac{\phi(\phi-1)}{r(x)^{2}}
$$

where $K(P)$ is the sectional curvature of any plane $P \subset T_{x} M$ that contains the radial vector field $\nabla r(x)$ and $x$ is any point in $M \backslash B\left(o, R_{0}\right)$. Then there exist $F, F_{1} \in \mathcal{F}$ such that

$$
F\left(\frac{r(x)}{j(x)}\right) j(x)^{C(n-1)} \leq r(x)^{-2}
$$

and

$$
F_{1}\left(\frac{r(x)^{2}}{j(x)^{2}}\right) j(x)^{C(n-1)} \leq r(x)^{-2}
$$

for any positive constant $C$ and for every $x \in M$ outside a compact set.

Proof. We prove the claim only for function $F$ since the case with $F_{1}$ (given by Proposition 2.5 is essentially the same. Let $\lambda$ be as in Proposition 2.5. By 2.5 there exists $F \in \mathcal{F}$ such that

$$
F(t) \leq \exp \left(-\frac{1}{t}\left(\log \left(e+\frac{1}{t}\right)\right)^{-\lambda}\right)
$$

for all small $t$. Hence the claim follows if

$$
\exp \left(-\frac{j(x)}{r(x)}\left(\log \left(e+\frac{j(x)}{r(x)}\right)\right)^{-\lambda}\right) j(x)^{C(n-1)} \leq r(x)^{-2},
$$


and taking logarithms, we see that this is equivalent with

$$
\frac{j(x)}{r(x)}\left(\log \left(e+\frac{j(x)}{r(x)}\right)\right)^{-\lambda}-C(n-1) \log j(x)-2 \log r(x) \geq 0 .
$$

It follows from the curvature assumptions that $j(x) \geq \operatorname{cr}(x)^{\phi}, \phi>1$, whenever $r(x) \geq \tilde{R}$ for some $\tilde{R}>0$ (see e.g. Lemma 2.1 and [30, Example 1]), so it is enough to show that

$$
f(t):=\frac{t}{a}\left(\log \left(e+\frac{t}{a}\right)\right)^{-\lambda}-C(n-1) \log t-2 \log a \geq 0
$$

for all $t \geq c a^{\phi}$ when $a$ is big enough. A straightforward computation gives that

$$
f^{\prime}(t)=\frac{\frac{1}{a}\left(1-\frac{\lambda}{\log (e+t / a)(a e / t+1)}\right)}{\left(\log \left(e+\frac{t}{a}\right)\right)^{\lambda}}-\frac{C(n-1)}{t},
$$

so noticing that $t / a \geq c a^{\phi-1} \geq \tilde{R}^{\phi}$ and $\log (e+t / a) \leq k(t / a)^{\alpha}$, where $k$ is a constant and $\alpha>0$ can be made as small as we wish, we obtain

$$
f^{\prime}(t) \geq \frac{k}{a^{1-\alpha} t^{\alpha}}-\frac{C(n-1)}{t} \geq 0
$$

for all $t \geq c a^{\phi}$ and $a$ large enough. Finally we notice that

$$
\begin{aligned}
f\left(a^{\phi}\right) & =a^{\phi-1}\left(\log \left(e+a^{\phi-1}\right)\right)^{-\lambda}-C(n-1) \log a^{\phi-1}-2 \log a \\
& =a^{\phi-1}\left(\log \left(e+a^{\phi-1}\right)\right)^{-\lambda}-(C(n-1)(\phi-1)+2) \log a
\end{aligned}
$$

which clearly is positive when $a \geq \tilde{R}$ is large enough.

3.1. Solving the asymptotic Dirichlet problem with Lipschitz boundary data. In order to prove the main theorem we begin by solving the corresponding Dirichlet problem with Lipschitz boundary data. The asymptotic boundary $\partial_{\infty} M$ is homeomorphic to the unit sphere $\mathbb{S}^{n-1} \subset T_{o} M$ and hence we may interpret the given boundary function $f \in C\left(\partial_{\infty} M\right)$ as a continuous function on $\mathbb{S}^{n-1}$. We first solve the asymptotic Dirichlet problem for 1.1) with Lipschitz continuous boundary values $f \in C\left(\mathbb{S}^{n-1}\right)$. We assume that, for all $x \in M$ and for all 2-planes $P \subset T_{x} M$,

$$
K(P) \leq-a^{2}(r(x))
$$

where $a:[0, \infty) \rightarrow[0, \infty)$ is a smooth function that is constant in some neighborhood of 0 and

$$
a^{2}(t)=\frac{\phi(\phi-1)}{t^{2}}, \quad \phi>1,
$$

for $t \geq R_{0}$. Identify $\partial_{\infty} M$ with the unit sphere $\mathbb{S}^{n-1} \subset T_{o} M$ and assume that $f: \mathbb{S}^{n-1} \rightarrow \mathbb{R}$ is $L$-Lipschitz. We extend $f$ radially to a continuous function $\theta$ on $M \backslash\{o\}$. The radial extension $\theta$ is also a locally Lipschitz function and hence, by Rademacher's theorem, differentiable almost everywhere. The gradient of $\theta$ can be estimated in terms of an angle function as follows. Let $x, y \in \bar{M}$ and let $\gamma^{o, x}$ and $\gamma^{o, y}$ be the unique unit speed geodesics joining $o$ to $x$ and $y$. Denote by $\bar{x}$ and $\bar{y}$ the corresponding points on $\mathbb{S}^{n-1}$ i.e. $\bar{x}=\dot{\gamma}_{0}^{o, x}$ and $\bar{y}=\dot{\gamma}_{0}^{o, y}$. Then

$$
\begin{aligned}
\frac{|\theta(x)-\theta(y)|}{d(x, y)} & =\frac{|\theta(\bar{x})-\theta(\bar{y})|}{d(x, y)} \leq \frac{L d(\bar{x}, \bar{y})}{d(x, y)} \\
& =L \frac{\varangle_{o}(\bar{x}, \bar{y})}{d(x, y)}=L \frac{\varangle_{o}(x, y)}{d(x, y)}
\end{aligned}
$$


and we obtain $|\nabla \theta| \leq L\left|\nabla \varangle_{o}(\cdot, \cdot)\right|$. By Lemma 2.3 this implies

$$
|\nabla \theta(x)| \leq \frac{L}{j(x)}
$$

and we see that $\theta$ satisfies the assumptions of Lemmas 3.3 and 3.4 .

We are now ready to solve the asymptotic Dirichlet problem with Lipschitz boundary data.

Lemma 3.7. Let $M$ be a Cartan-Hadamard manifold of dimension $n \geq 2$ satisfying the curvature assumptions 1.4, 1.5 and (1.6) for all 2-planes $P \subset T_{x} M$ with $x \in M \backslash B\left(o, R_{0}\right)$. Suppose that $f \in C\left(\partial_{\infty} M\right)$ is L-Lipschitz when interpreted as a function on $\mathbb{S}^{n-1} \subset T_{o} M$. Then the asymptotic Dirichlet problem for minimal graph equation (1.1) is uniquely solvable with boundary data $f$.

Proof. Let $\theta$ be the radial extension of the given Lipschitz boundary data $f \in$ $C\left(\partial_{\infty} M\right)$ defined above. We exhaust $M$ by an increasing sequence of geodesic balls $B_{k}=B(o, k), k \in \mathbb{N}$, and show first that there exist smooth solutions $u_{k} \in$ $C^{\infty}\left(B_{k}\right) \cap C\left(\bar{B}_{k}\right)$ of the minimal graph equation

$$
\left\{\begin{array}{l}
\operatorname{div} \frac{\nabla u_{k}}{\sqrt{1+\left|\nabla u_{k}\right|^{2}}}=0, \quad \text { in } B_{k} \\
u_{k}\left|\partial B_{k}=\theta\right| \partial B_{k} .
\end{array}\right.
$$

For this, fix $k \in \mathbb{N}$ and let $\left(\theta_{i}^{k}\right) \subset C^{2}\left(\partial B_{k}\right)$ be a sequence that converges uniformly to the function $\theta$ on $\partial B_{k}$. For every $i$ there exists a function $u_{i}^{k} \in C^{\infty}\left(B_{k}\right)$ that solves the minimal graph equation in $B_{k}$ and has boundary values $\theta_{i}^{k}$. By the Maximum principle we have

$$
\sup _{B_{k}}\left|u_{j}^{k}-u_{i}^{k}\right| \leq \sup _{\partial B_{k}}\left|\theta_{j}^{k}-\theta_{i}^{k}\right|
$$

so the sequence $\left(u_{i}^{k}\right)$ converges uniformly to some function $u_{k} \in C\left(\bar{B}_{k}\right)$. In $\bar{B}_{k}$ the sectional curvatures are bounded, so we can apply the interior gradient estimate 28 , Theorem 1.1] and obtain that $\left|\nabla u_{i}^{k}\right|$ is locally bounded independent of $i$. Therefore standard arguments and regularity theory of elliptic PDEs imply that $u_{i}^{k} \rightarrow u_{k}$ in $C_{\text {loc }}^{2}\left(B_{k}\right) \cap C\left(\bar{B}_{k}\right)$ and therefore $u_{k}$ is also a solution to the minimal graph equation (3.11). Moreover, the comparison principle implies that

$$
-\max _{x \in M}|\theta(x)| \leq u_{k} \leq \max _{x \in M}|\theta(x)|,
$$

so the solutions $u_{k}$ are bounded in $B_{k}$ for every $k \in \mathbb{N}$.

Fix a compact set $K \subset M$. Then applying the interior gradient estimate [28, Theorem 1.1], we obtain

$$
\sup _{K}\left|\nabla u_{k}\right| \leq c(K)
$$

where the constant $c(K)$ is independent of $k$. The theory of elliptic PDEs implies that there exists a subsequence, still denoted by $u_{k}$, that converges in $C_{\text {loc }}^{2}(M)$ to a solution $u \in C^{\infty}(M)$. Hence we are left to prove that $u$ extends continuously to the boundary $\partial_{\infty} M$ and satisfies $u \mid \partial_{\infty} M=f$.

Next we will use Lemma 3.3 , and in order to estimate the appearing integrals we use geodesic polar coordinates $(r, v)$ for points $x \in M$. Here we denoted $r=r(x)$ and $v=\dot{\gamma}_{0}^{o, x} \in S_{o} M$. Let $\lambda(r, v)$ be the Jacobian for these polar coordinates. Note that then we have $\lambda(r, v) \leq J(r, v)^{n-1}$ where $J(x)$ denotes the supremum of $|V(r(x))|$ over Jacobi fields $\mathrm{V}$ along the geodesic $\gamma^{o, x}$ that satisfy $V_{0}=0,\left|V_{0}^{\prime}\right|=1$ and $V_{0}^{\prime} \perp \dot{\gamma}_{0}^{o, x}$. 
Let $\nu$ be such that it satisfies the assuptions of Lemmas 3.3 and 3.4. Applying Lemma 2.2. Fatou's lemma, and Lemma 3.3 with $U=B_{k}$ we get

$$
\begin{aligned}
\int_{M} \varphi(|u-\theta| / \nu) \leq \liminf _{k \rightarrow \infty} \int_{B_{k}} \varphi\left(\left|u_{k}-\theta\right| / \nu\right) \\
\leq c+c \int_{M} F(r|\nabla \theta|)+c \int_{M} F_{1}\left(r^{2}|\nabla \theta|^{2}\right) \\
=c+c \int_{R_{1}}^{\infty} \int_{S_{o} M} F(r|\nabla \theta(r, v)|) \lambda(r, v) d v d r \\
\quad+c \int_{R_{1}}^{\infty} \int_{S_{o} M} F_{1}\left(r^{2}|\nabla \theta(r, v)|^{2}\right) \lambda(r, v) d v d r \\
\leq c+c \int_{R_{1}}^{\infty} \int_{S_{o} M} F\left(\frac{r}{j(r, v)}\right) j(r, v)^{C_{K}(n-1)} d v d r \\
\quad+c \int_{R_{1}}^{\infty} \int_{S_{o} M} F_{1}\left(\frac{r^{2}}{j(r, v)^{2}}\right) j(r, v)^{C_{K}(n-1)} d v d r \\
<\infty
\end{aligned}
$$

Finiteness of the last integrals follows from Lemma 3.6

Let $x \in M$ and fix $s \in\left(0, r_{S}\right)$. For $k$ large enough, $u_{k}$ satisfies the assumptions of Lemma 3.4 , and hence

$$
\sup _{B(x, s / 2)} \varphi\left(\left|u_{k}-\theta\right| / \nu\right)^{n+1} \leq c \int_{B(x, s)} \varphi\left(\left|u_{k}-\theta\right| / \nu\right) .
$$

This and the dominated convergence theorem implies that

$$
\begin{aligned}
\sup _{B(x, s / 2)} & \varphi(|u-\theta| / \nu)^{n+1}=\sup _{B(x, s / 2)} \lim _{k \rightarrow \infty} \varphi\left(\left|u_{k}-\theta\right| / \nu\right)^{n+1} \\
& \leq \limsup _{k \rightarrow \infty} \sup _{B(x, s / 2)} \varphi\left(\left|u_{k}-\theta\right| / \nu\right)^{n+1} \\
& \leq c \limsup _{k \rightarrow \infty} \int_{B(x, s)} \varphi\left(\left|u_{k}-\theta\right| / \nu\right)=c \int_{B(x, s)} \varphi(|u-\theta| / \nu) .
\end{aligned}
$$

Let $\xi \in \partial_{\infty} M$ and $\left(x_{i}\right)$ be a sequence of points in $M$ with $x_{i} \rightarrow \xi$ as $i \rightarrow \infty$. Applying the estimate 3.13 with $x=x_{i}$ and fixed $s \in\left(0, r_{S}\right)$ we obtain, by 3.12 , that

$$
\lim _{i \rightarrow \infty} \sup _{B\left(x_{i}, s / 2\right)} \varphi(|u-\theta| / \nu)^{n+1} \leq c \lim _{i \rightarrow \infty} \int_{B\left(x_{i}, s\right)} \varphi(|u-\theta| / \nu)=0
$$

and hence $\left|u\left(x_{i}\right)-\theta\left(x_{i}\right)\right| \rightarrow 0$ as $i \rightarrow \infty$. Since $\xi \in \partial_{\infty} M$ was arbitrary, it follows that $u$ extends continuously to $\partial_{\infty} M$ and satisfies $u \mid \partial_{\infty} M=f$.

3.2. Proof of the main theorem. Let $f \in C\left(\partial_{\infty} M\right)$. As in the case of Lipschitz functions, we identify $\partial_{\infty} M$ with the unit sphere $\mathbb{S}^{n-1} \subset T_{o} M$. Let $\left(f_{i}\right)$ be a sequence of Lipschitz functions such that $f_{i} \rightarrow f$ uniformly as $i \rightarrow \infty$. By Lemma 3.7 there exist solutions $u_{i} \in C^{\infty}(M) \cap C(\bar{M})$ of the minimal graph equation (1.1) with the desired boundary values $u_{i}=f_{i}$ on $\partial_{\infty} M$. It follows from the Maximum principle that

$$
\sup _{M}\left|u_{i}-u_{j}\right|=\max _{\partial_{\infty} M}\left|f_{i}-f_{j}\right|
$$

and consequently the sequence $u_{i}$ converges uniformly to a function $u \in C(\bar{M})$. Applying the interior gradient estimate [28, Theorem 1.1] in compact subsets of $M$ we conclude that the convergence takes place in $C(\bar{M}) \cap C_{\text {loc }}^{2}(M)$ and therefore $u$ is also a solution to 11.1 in $M$ and $u=f$ on $\partial_{\infty} M$. Regularity theory implies that $u \in C^{\infty}(M)$. 
For the proof of uniqueness, suppose that $u$ and $v$ are both solutions of (1.1) in $M$, continuous in $\bar{M}$ and $u=v$ on the boundary $\partial_{\infty} M$. By symmetry we can assume that $u(y)>v(y)$ for some $y \in M$. Denote $\delta=(u(y)-v(y)) / 2$ and let $U \subset\{x \in M: u(x)>v(x)+\delta\}$ be the component that contains $y$. Then $U$ is a relatively compact open domain since both $u$ and $v$ are continuous and coincide on $\partial_{\infty} M$. Furthemore $u=v+\delta$ on $\partial U$ and it follows that $u=v+\delta$ in $U$ which is a contradiction since we have $y \in U$.

\section{REFERENCES}

[1] M. T. Anderson. The Dirichlet problem at infinity for manifolds of negative curvature. $J$. Differential Geom., 18(4):701-721 (1984), 1983.

[2] A. Borbély. The nonsolvability of the Dirichlet problem on negatively curved manifolds. Differential Geom. Appl., 8(3):217-237, 1998.

[3] J.-B. Casteras, I. Holopainen, and J.B. Ripoll. On the asymptotic Dirichlet problem for the minimal hypersurface equation in a Hadamard manifold. preprint, 2013. arXiv: 1311.5693.

[4] J.-B. Casteras, I. Holopainen, and J.B. Ripoll. Asymptotic Dirichlet problem for $\mathcal{A}$ harmonic and minimal graph equations in Cartan-Hadamard manifolds. preprint, 2015. arXiv: 1501.05249 .

[5] J. Cheeger, M. Gromov, and M. Taylor. Finite propagation speed, kernel estimates for functions of the Laplace operator, and the geometry of complete Riemannian manifolds. J. Differential Geom., 17(1):15-53, 1982.

[6] S. Y. Cheng. The Dirichlet problem at infinity for non-positively curved manifolds. Comm. Anal. Geom., 1(1):101-112, 1993.

[7] H. I. Choi. Asymptotic Dirichlet problems for harmonic functions on Riemannian manifolds. Trans. Amer. Math. Soc., 281(2):691-716, 1984.

[8] P. Collin and H. Rosenberg. Construction of harmonic diffeomorphisms and minimal graphs. Ann. of Math. (2), 172(3):1879-1906, 2010.

[9] C. B. Croke. Some isoperimetric inequalities and eigenvalue estimates. Ann. Sci. École Norm. Sup. (4), 13(4):419-435, 1980.

[10] M. Dajczer and J. H. de Lira. Killing graphs with prescribed mean curvature and Riemannian submersions. Ann. Inst. H. Poincaré Anal. Non Linéaire, 26(3):763-775, 2009.

[11] M. Dajczer and J. H. S. de Lira. Entire bounded constant mean curvature Killing graphs. J. Math. Pures Appl. (9), 103(1):219-227, 2015.

[12] M. Dajczer, P. A. Hinojosa, and J. H. de Lira. Killing graphs with prescribed mean curvature. Calc. Var. Partial Differential Equations, 33(2):231-248, 2008.

[13] Q. Ding, J. Jost, and Y. Xin. Minimal graphic functions on manifolds of non-negative Ricci curvature. Preprint arXiv:1310.2048v2, 2013.

[14] N. do Espírito-Santo, S. Fornari, and J. B. Ripoll. The Dirichlet problem for the minimal hypersurface equation in $M \times \mathbb{R}$ with prescribed asymptotic boundary. J. Math. Pures Appl. (9), 93(2):204-221, 2010.

[15] N. do Espírito-Santo and J. Ripoll. Some existence results on the exterior Dirichlet problem for the minimal hypersurface equation. Ann. Inst. H. Poincaré Anal. Non Linéaire, 28(3):385393, 2011.

[16] P. Eberlein and B. O'Neill. Visibility manifolds. Pacific J. Math., 46:45-109, 1973.

[17] J. A. Gálvez and H. Rosenberg. Minimal surfaces and harmonic diffeomorphisms from the complex plane onto certain Hadamard surfaces. Amer. J. Math., 132(5):1249-1273, 2010.

[18] R. E. Greene and H. Wu. Function theory on manifolds which possess a pole, volume 699 of Lecture Notes in Mathematics. Springer, Berlin, 1979.

[19] B. Guan and J. Spruck. Hypersurfaces of constant mean curvature in hyperbolic space with prescribed asymptotic boundary at infinity. Amer. J. Math., 122(5):1039-1060, 2000.

[20] B. Guan, J. Spruck, and L. Xiao. Interior curvature estimates and the asymptotic plateau problem in hyperbolic space. J. Differential Geom., 96(2):201-222, 2014.

[21] I. Holopainen. Asymptotic Dirichlet problem for the $p$-Laplacian on Cartan-Hadamard manifolds. Proc. Amer. Math. Soc., 130(11):3393-3400 (electronic), 2002.

[22] I. Holopainen and J. Ripoll. Nonsolvability of the asymptotic Dirichlet problem for some quasilinear elliptic PDEs on Hadamard manifolds. Rev. Mat. Iberoam., 31(3):1107-1129, 2015.

[23] I. Holopainen and A. Vähäkangas. Asymptotic Dirichlet problem on negatively curved spaces. J. Anal., 15:63-110, 2007. 
[24] A. Kufner, O. John, and S. Fučík. Function spaces. Noordhoff International Publishing, Leyden; Academia, Prague, 1977. Monographs and Textbooks on Mechanics of Solids and Fluids; Mechanics: Analysis.

[25] J. Ripoll and M. Telichevesky. Regularity at infinity of Hadamard manifolds with respect to some elliptic operators and applications to asymptotic Dirichlet problems. Trans. Amer. Math. Soc., 367(3):1523-1541, 2015.

[26] H. Rosenberg, F. Schulze, and J. Spruck. The half-space property and entire positive minimal graphs in $M \times \mathbb{R}$. J. Differential Geom., 95(2):321-336, 2013.

[27] R. Schoen and S. T. Yau. Lectures on harmonic maps. Conference Proceedings and Lecture Notes in Geometry and Topology, II. International Press, Cambridge, MA, 1997.

[28] J. Spruck. Interior gradient estimates and existence theorems for constant mean curvature graphs in $M^{n} \times \mathbf{R}$. Pure Appl. Math. Q., 3(3, Special Issue: In honor of Leon Simon. Part 2):785-800, 2007.

[29] D. Sullivan. The Dirichlet problem at infinity for a negatively curved manifold. J. Differential Geom., 18(4):723-732 (1984), 1983.

[30] A. Vähäkangas. Dirichlet problem at infinity for $\mathcal{A}$-harmonic functions. Potential Anal., 27(1):27-44, 2007.

[31] A. Vähäkangas. Dirichlet problem on unbounded domains and at infinity. Reports in Mathematics, Preprint 499, Department of Mathematics and Statistics, University of Helsinki, 2009.

J.-B. Casteras, Departement de Mathematique Universite libre de Bruxelles, CP 214, Boulevard du Triomphe, B-1050 Bruxelles, Belgium

E-mail address: jeanbaptiste.casteras@gmail.com

E. Heinonen, Department of Mathematics and Statistics, P.O.B. 68 (Gustaf HälSTRÖMin KATU 2B), 00014 University of Helsinki, Finland.

E-mail address: esko.heinonen@helsinki.fi

I.Holopainen, Department of Mathematics and Statistics, P.O.B. 68 (Gustaf HälSTRÖMIN KATU 2B), 00014 University OF Helsinki, Finland.

E-mail address: ilkka.holopainen@helsinki.fi 